\title{
ON A CLASS OF PERTURBATIONS OF THE HARMONIC OSCILLATOR ${ }^{1}$
}

\author{
PHILIP HARTMAN
}

1. The following theorem, concerning solutions of

$$
y^{\prime \prime}+[1+f(x)+h(x) \cos 2 \eta x] y=0,
$$

was proved by Atkinson in the cases $\alpha=1$ (where the sum in (1.6) is empty) and $\alpha=2$; see [1, p. 349 and p. 355]. In [3], Kelman and Madsen formulated the general result $(\alpha=1,2, \cdots)$ and proved it using different methods.

THEOREM 1.1 [3]. Let $f(x) \in L^{1}[0, \infty) ; h(x)$ of bounded variation on $[0, \infty)$ for which there exists an integer $\alpha>0$ satisfying

$$
\int^{\infty}|h|^{\alpha} d x=\infty \text { and } \int^{\infty}|h|^{\alpha+1} d x<\infty ;
$$

$\eta>0$ a constant satisfying

(1.3) $0<\eta \neq k / j$, where $1 \leqq k \leqq \alpha-1$ and $1 \leqq j \leqq \alpha$, and, if $\alpha$ is odd,

$$
0<\eta \neq \alpha / j \quad \text { for } j=1,3, \cdots, \alpha .
$$

Then for even integers $2 j, 2 \leqq 2 j \leqq \alpha$, there are real-valued rational functions $c_{2 j}=c_{2 j}(\eta)$ of $\eta$ finite on (1.3)-(1.4), with the following property: There exists a one-to-one correspondence between solutions $y(x)$ of (1.1) and pairs of constants $\left(a_{1}, a_{2}\right)$ such that

$$
\begin{aligned}
y & =a_{1} \sin \theta(x)+a_{2} \cos \theta(x)+o(1), \\
y^{\prime} & =a_{1} \cos \theta(x)-a_{2} \sin \theta(x)+o(1), \\
\theta(x) & =x+\sum_{2 \leq 2 j \leqslant \alpha} c_{2 j} \int_{0}^{x} h^{2 j}(s) d s .
\end{aligned}
$$

For related (less precise) results, see references in [3] to J. G. van der Corput.

Using a device from Hartman [2], we shall give a somewhat more

Received by the editors September 29, 1966.

1 This research was supported by the Air Force Office of Scientific Research, Office of Aerospace Research, United States Air Force, under AFOSR Contract No. F 44620-67-C-0098. 
transparent proof and, at the same time, replace (1.1) by the more general equation

$$
y^{\prime \prime}+\left[1+2 f(x)+2 \sum_{m=0}^{M} h_{m}(x) \cos \left(2 \eta_{m} x+\gamma_{m}\right)\right] y=0 .
$$

It should be pointed out that Atkinson [1] had used a related method for obtaining Theorem 1.1 for $\alpha=2$ and had noted that this argument can be used to show the validity of the following result for $\alpha=2$, $h_{0} \equiv 0, \gamma_{1}=\gamma_{2}=\cdots=\gamma_{M}=0$.

TheOREM 1.2. Let $f(x) \in L^{1}[0, \infty) ; h_{0}(x), \cdots, h_{M}(x)$ functions of bounded variation on $[0, \infty)$ for which there is an integer $\alpha>0$ satisfying

$$
\sum_{m=0}^{M} \int^{\infty}\left|h_{M}\right|^{\alpha+1} d x<\infty ;
$$

let $\eta_{0}=0<\eta_{1} \leqq \cdots \leqq \eta_{m}$ be constants with the property that

$$
\text { (1.9) }\left|\eta_{m(1)} \pm \eta_{m(2)} \pm \cdots \pm \eta_{m(\nu)}\right| \neq \tau, \quad \text { where } \tau=1, \cdots, \nu
$$

whenever

$$
0 \leqq m(j) \leqq M, \quad 1 \leqq \nu \leqq \alpha, \quad \int^{\infty} \prod_{j=1}^{\nu}\left|h_{m(j)}\right| d x=\infty ;
$$

finally, $\gamma_{0}=0$ and $\gamma_{1}, \cdots, \gamma_{M}$ are arbitrary constants. Then there exists $a$ one-to-one correspondence between solutions $y(x)$ of (1.7) and pairs of constants $\left(a_{1}, a_{2}\right)$ such that

$$
\begin{aligned}
y= & a_{1} \sin \theta(x)+a_{2} \cos \theta(x)+o(1), \\
y^{\prime}= & a_{1} \cos \theta(x)-a_{2} \sin \theta(x)+o(1), \\
\theta(x)= & x+\int_{0}^{x} h_{0} d s \\
& +\sum_{\mu=2}^{\alpha} \sum_{I[\mu]} c_{I[\mu]}\left(\cos \Gamma_{I[\mu]}\right) \int_{0}^{x} \prod_{j=1}^{\mu} h_{m(j)} d s, \\
I[\mu]= & (m(1), \pm m(2), \cdots, \pm m(\mu)), \\
\Gamma_{I[\mu]}= & \gamma_{m(1)} \pm \gamma_{m(2)} \pm \cdots \pm \gamma_{m(\mu)},
\end{aligned}
$$

$c_{I[\mu]}=c_{m(1), \pm m(2)}, \cdots, \pm m(\mu)$ are rational functions of $\left(\eta_{m(1)}, \cdots, \eta_{m(\mu)}\right)$ which are finite for (1.9), and $\sum_{I[\mu]}$ is the sum over the set of indices $I[\mu]$ $=(m(1), \pm m(2), \cdots, \pm m(\mu))$ for which $0 \leqq m(j) \leqq M$, 


$$
\eta_{m(1)} \pm \eta_{m(2)} \pm \cdots \pm \eta_{m(\mu)}=0 \text { and }
$$

$$
\int_{0}^{\infty} \prod_{j=1}^{\mu} h_{m(j)} d s \text { is not convergent. }
$$

-REMARK 1. The rational functions $c_{m(1), \pm m(2)}, \cdots, \pm m(\mu)$ are independent of the solution $y(x)$, of the function $f(x)$, and of the functions $\left(h_{0}, \cdots, h_{M}\right)$ within the class of sets of functions $\left(h_{0}, \cdots, h_{M}\right)$ for which the convergence properties of the integrals $\int^{\infty} \prod h_{m(j)} d x$ do not vary. Note that if $\mu=1$, then (1.12) can hold only for $m(1)=0$.

REMARK 2. If, for some $k$ on $0 \leqq k \leqq M, h_{k}(x) \equiv 0$ or more generally, $\int^{\infty}\left|h_{k}\right| d x<\infty$, then the corresponding term $2 h_{k}(x) \cos \left(2 \eta_{k} x+\gamma_{k}\right)$ in (1.7) can be considered part of the term $2 f(x)$. In this case, no $m(j)=k$ occurs in (1.9), (1.11), and (1.12).

REMARK 3. In the special case (1.1) of (1.7), we have $h_{0}(x) \equiv 0$, $\eta_{m(j)}=\eta$ for all $j \geqq 1$, and (1.9) is equivalent to (1.3)-(1.4). Also the first part of (1.12) cannot hold unless $\mu=2 j$ is even (and there are $j$ signs + and $j$ signs -$)$, so that (1.10)-(1.11) reduce to (1.5)-(1.6). (In order to see the equivalence of (1.9) and (1.3)-(1.4), let $\mu$ be the number of + signs in $\left|+\eta_{m(1)} \pm \eta_{m(2)} \pm \cdots \pm \eta_{m(\nu)}\right|$, so that the conditions on $\eta$ become $0<|2 \mu-\nu| \eta \neq \tau$ for $\mu, \tau=1, \cdots, \nu$ and $\nu=1, \cdots, \alpha$. If $\alpha$ is odd, this reduces to (1.3)-(1.4). If $\alpha$ is even, this reduces to (1.3)-(1.4) and the apparently additional conditions $0<\eta \neq \alpha / j$ for $j=2,4, \cdots, \alpha$. But these additional conditions are contained in (1.3).

2. Proof of Theorem 1.2. Introduce the abbreviation

$$
F(x)=\sum_{m=0}^{M} h_{m}(x) \cos \left(2 \eta_{m} x+\gamma_{m}\right) .
$$

From the Prüfer transformation

$$
y(x)=r(x) \sin \phi(x), \quad y^{\prime}(x)=r(x) \cos \phi(x),
$$

and (1.7), we get

(2.2) $\quad d \log r=-F(x) \sin 2 \phi d x-f(x) \sin 2 \phi d x$,

$$
d \phi=d x+F(x)(1-\cos 2 \phi) d x+f(x)(1-\cos 2 \phi) d x .
$$

Following a device of Hartman [2], the last relation will also be used in the form

(2.4) $d s=d \phi(s)-f(s)(1-\cos 2 \phi(s)) d s-F(s)(1-\cos 2 \phi(s)) d s$.

In view of (2.2), 
(2.5) $\log r(x)=c+o(1)+\sum_{m=0}^{M} \int_{0}^{x} h_{m} \cos \left(2 \eta_{m} s+\gamma_{m}\right) \sin 2 \phi(s) d s ;$ also, we have

$$
\phi(x)=x+c+o(1)+\int_{0}^{x} F d s-\int_{0}^{x} F \cos 2 \phi d s .
$$

Since $h_{m}$ is of bounded variation on $[0, \infty)$,

$$
\int_{0}^{\infty} h_{m}(x) \cos \left(2 \eta_{m} x+\gamma_{m}\right) d x=\lim _{T \rightarrow \infty} \int_{0}^{T} \quad \text { exists if } \eta_{m} \neq 0 .
$$

Thus

$$
\begin{aligned}
\phi(x)= & x+c+o(1)+\int_{0}^{x} h_{0} d s \\
& -\sum_{m=0}^{M} \int_{0}^{x} h_{m} \cos \left(2 \eta_{m} s+\gamma_{m}\right) \cos 2 \phi d s .
\end{aligned}
$$

In (2.5), (2.7) and below, $c$ will always denote a constant not necessarily the same one. The analogue of (2.6) will be used repeatedly below.

LEMMA 2.1. Let $\phi(x)$ be as above; $g(x)$ a function of bounded variation on $[0, \infty), g(x)=o(1)$ as $x \rightarrow \infty ; \sigma, \tau, \gamma^{0}, \gamma$ and $\delta$ real constants such that

$$
|\sigma| \neq|\tau|, \quad \tau \neq 0 .
$$

Then, as $x \rightarrow \infty$,

$$
\begin{array}{r}
\int_{0}^{x} g(s) \cos \left(2 \sigma s+\gamma^{0}-\gamma\right) \cos (2 \tau \phi-\delta) d s \\
=c+o(1)+4\left(\tau^{2}-\sigma^{2}\right)^{-1}\{\cdots\},
\end{array}
$$

where $\{\cdots\}$ is the expression

$$
\begin{aligned}
\{\cdots\} & =(\sigma \tau) \sum_{m=0}^{M} \sum_{j=0}^{1} \sum_{k=-1}^{1} \epsilon_{k} \int_{0}^{x} g h_{m} \\
& \times \sin \left[2\left(\sigma+(-1)^{j} \eta_{m}\right) s+\gamma^{0}-\gamma+(-1)^{j} \gamma_{m}\right] \\
& \times \sin [2(\tau+k) \phi-\delta] d s+\tau^{2} \sum_{m=0}^{M} \sum_{j=0}^{1} \sum_{k=-1}^{1} \epsilon_{k} \int_{0}^{x} g h_{m} \\
& \times \cos \left[2\left(\sigma+(-1)^{j} \eta_{m}\right) s+\gamma^{0}-\gamma+(-1)^{j} \gamma_{m}\right] \\
& \times \cos [2(\tau+k) \phi-\delta] d s
\end{aligned}
$$


$\epsilon_{ \pm 1}=1$ and $\epsilon_{0}=-2$.

We shall only need the cases $\gamma=\delta=0$ and $\gamma=\delta=\pi / 2$ for the asymptotic behavior of $\phi(x)$, and the cases $\gamma=\pi / 2, \delta=0$ and $\gamma=0, \delta=\pi / 2$ for $r(x)$.

Proof. Let $I$ denote the integral on the left of (2.9). Replace $d s$ in $I$ by its value in (2.4) and integrate the resulting first term by parts to obtain

$$
I=c+o(1)+(\sigma / \tau) \int_{0}^{x} g(s) \sin \left(2 \sigma s+\gamma^{0}-\gamma\right) \sin (2 \tau \phi-\delta) d s
$$

$$
-\int_{0}^{x} g(s) \cos \left(2 \sigma s+\gamma^{0}-\gamma\right) \cos (2 \tau \phi-\delta) F(s)(1-\cos 2 \phi) d s .
$$

Use (2.4) in the first integral on the right of (2.11) and integrate the first term by parts,

$$
\begin{aligned}
I= & c+o(1)+(\sigma / \tau)^{2} I \\
& -(\sigma / \tau) \int_{0}^{x} g(s) \sin \left(2 \sigma s+\gamma^{0}-\gamma\right) \sin (2 \tau \phi-\delta) F(s)(1-\cos 2 \phi) d s \\
& -\int_{0}^{x} g(s) \cos \left(2 \sigma s+\gamma^{0}-\gamma\right) \cos (2 \tau \phi-\delta) F(s)(1-\cos 2 \phi) d s .
\end{aligned}
$$

In view of the relations, for $\chi=\sin$ or $\chi=\cos$,

$$
\begin{aligned}
2 \chi(2 \tau \phi & -\delta)(1-\cos 2 \phi) \\
& =-\sum_{k=-1}^{1} \epsilon_{k} \chi[2(\tau+k) \phi-\delta],
\end{aligned}
$$

and

$$
\begin{aligned}
& 2 \chi\left(2 \sigma s+\gamma^{0}-\gamma\right) F(s) \\
& \quad=\sum_{m=0}^{M} \sum_{j=0}^{1} h_{m}(s) \chi\left[2\left(\sigma+(-1)^{j} \eta_{m}\right) s+\gamma^{0}-\gamma+(-1)^{j} \gamma_{m}\right],
\end{aligned}
$$

formula (2.9) follows. This completes the proof.

On $\phi(x)$. We now show, by an induction on $\nu$ for $1 \leqq \nu \leqq \alpha+1$, that, under the assumption (1.9), $\phi(x)$ has the form 


$$
\begin{aligned}
\phi(x)=x & +c+o(1)+\int_{0}^{x} h_{0} d s \\
& +\sum_{\mu=1}^{\nu-1} \sum_{I[\mu]} c_{I[\mu]}\left(\cos \Gamma_{I[\mu]}\right) \int_{0}^{x} \prod_{j=1}^{\mu} h_{m(j)} d s \\
& +\sum_{\tau=1}^{\nu} \sum_{I[\mu]}^{\prime} a_{\tau, I[\nu]} \int_{0}^{x} \prod_{j=1}^{\nu} h_{m(j)} \\
& \times \cos \left(2 N_{I[\nu]} s+\Gamma_{I[\nu]}\right) \cos 2 \tau \phi d s \\
& +\sum_{\tau=1}^{\nu} \sum_{I[\nu]}^{\prime} b_{\tau, I[\nu]} \int_{0}^{x} \prod_{j=1}^{\nu} h_{m(j)} \\
& \times \sin \left(2 V_{I[\nu]} s+\Gamma_{I[\nu]}\right) \sin 2 \tau \phi d s,
\end{aligned}
$$

where

$$
N_{I[\nu]}=\eta_{m(1)} \pm \eta_{m(2)} \pm \cdots \pm \eta_{m(\nu)}
$$

$c_{I[\mu]}=c_{m(1), \pm m[2], \cdots, \pm m(\mu)} \quad$ and $\quad a_{\tau, I[\nu]}=a_{\tau, m(1), \pm m(2), \cdots, \pm m[\nu]}, \quad b_{\tau, I[\nu]}$ $=b_{\tau, m(1), \pm m(2)}, \cdots, \pm m(\nu)$ are rational functions of $\left(\eta_{m(1)}, \cdots, \eta_{m(\mu)}\right)$ and of $\left(\eta_{m(1)}, \cdots, \eta_{m(\nu)}\right)$, respectively, finite for $(1.9) ; \sum_{I[\mu]}$ is the sum over the sets of indices $(m(1), \pm m(2), \cdots, \pm m(\mu)), 0 \leqq m(j) \leqq M$, for which

$$
\eta_{m(1)} \pm \cdots \pm \eta_{m(\mu)}=0 \text { and } \int^{\infty} \prod_{j=1}^{\mu} h_{m(j)} d x \text { is not convergent; }
$$

finally $\sum_{I[\nu]}^{\prime}$ is the sum over all sets $I[\nu]=(m(1), \pm m(2), \cdots$, $\pm m(\nu))$ for which

$$
\int \prod_{j=1}^{\nu}\left|h_{m(j)}\right| d x=\infty
$$

The formula (2.7) can be written in the form (2.12) for $\nu=1$. We assume (2.12) for some given $\nu, 1 \leqq \nu \leqq \alpha$. Then the assumption (1.9) makes Lemma 2.1 applicable to each term in the last two sums of (2.12), with $\gamma=\delta=\pi / 2$ in the last sum and $\gamma=\delta=0$ in the next to last sum, $\quad \sigma=\eta_{m(1)} \pm \cdots \pm \eta_{m(\nu)}, \quad \gamma^{0}=\gamma_{m(1)} \pm \cdots \pm \gamma_{m(\nu)}, \quad$ and $g(x)$ $=\prod h_{m(j)}$ for $j=1, \cdots, \nu$. This shows the validity of $(2.12)$ when $\nu$ is replaced by $\nu+1$, since

$$
\int_{0}^{x} \prod_{j=1}^{\nu+1} h_{m(j)} \cos \left(2 N_{I[\nu+1]} s+\Gamma_{I[\nu+1]}\right) d s=c+o(1)
$$


if the first part of (2.13) does not hold. Hence (2.12) is valid for $\nu=\alpha+1$. Thus $\phi(x)$ can be written in the form

$$
\phi(x)=c^{0}+o(1)+\theta(x),
$$

where $\theta(x)$ is independent of the solution $y(x)$ and is given by (1.11).

On $r(x)$. Starting with (2.5), the cases $\gamma=\pi / 2, \delta=0$ and $\gamma=0$, $\delta=\pi / 2$ of Lemma 2.1 imply, by an induction on $\nu, 1 \leqq \nu \leqq \alpha+1$, that $\log r(x)$ can be written in the form

$$
\begin{aligned}
\log r(x)= & c+o(1) \\
& +\sum_{\mu=1}^{\nu-1} \sum_{I[\mu]}^{*} c_{I[\mu]}^{*}\left(\sin \Gamma_{I[\mu]}\right) \int_{0}^{x} \prod_{j=1}^{\mu} h_{m(j)} d s \\
& +\sum_{\tau=1}^{\nu} \sum_{I[\nu]}^{\prime} a_{\tau, I[\nu]}^{*} \\
& \times \int_{0}^{x} \prod_{j=1}^{\nu} h_{m(j)} \cos \left(2 N_{I[\nu]} s+\Gamma_{I[\nu]}\right) \sin 2 \tau \phi d s \\
& +\sum_{\tau=1}^{\nu} \sum_{I[\nu]}^{\prime} b_{\tau, I[\nu]}^{*} \\
& \times \int_{0}^{x} \prod_{j=1}^{\nu} h_{m(j)} \sin \left(2 N_{I[\nu]} S+\Gamma_{I[\nu]}\right) \cos 2 \tau \phi d s
\end{aligned}
$$

in notation analogous to (2.12).

'Thus, the case $\nu=\alpha+1$ shows that

$$
r(x)=\left[c^{1}+o(1)\right] \exp \rho(x),
$$

where $\rho(x)$ is independent of the solution $y(x)$ and

$$
\rho(x)=\sum_{\mu=1}^{\alpha} \sum_{I[\mu]} c_{I[\mu]}^{*}\left(\sin \Gamma_{I[\mu]}\right) \int_{0}^{x} \prod_{j=1}^{\mu} h_{m(j)} d s .
$$

Completion of the Proof. Let $y_{1}(x), y_{2}(x)$ be two solutions of (1.7) with the Wronskian

$$
y_{1} y_{2}^{\prime}-y_{1}^{\prime} y_{2} \equiv 1 \text {. }
$$

Then, by (2.1), (2.15) and (2.17), for $j=1,2$,

$$
\begin{aligned}
y_{j} & =\left[c_{j}^{1}+o(1)\right] e^{\rho(x)} \sin \left[c_{j}^{0}+o(1)+\theta(x)\right], \\
y_{j}^{\prime} & =\left[c_{j}^{1}+o(1)\right] e^{\rho(x)} \cos \left[c_{j}^{0}+o(1)+\theta(x)\right],
\end{aligned}
$$


where $\left(c_{j}^{0}, c_{j}^{1}\right)$ are the constants $\left(c^{0}, c^{1}\right)$ belonging to $y_{j}(x)$. By (2.19),

$$
\left[c_{1}^{1} c_{2}^{1}+o(1)\right] e^{2 \rho(x)} \sin \left(c_{1}^{0}-c_{2}^{0}+o(1)\right) \equiv 1 .
$$

Thus, according as

$$
c_{1}^{1} c_{2}^{1} \sin \left(c_{1}^{0}-c_{2}^{0}\right)=0 \text { or } \neq 0,
$$

it follows that

$$
\lim _{x \rightarrow \infty} \rho(x)=+\infty \quad \text { or exists (finite). }
$$

Actually, the first alternative in (2.22) cannot hold. In order to see this, consider the differential equation obtained by changing the signs of the $\gamma_{m}$ in (1.7),

$$
y^{\prime \prime}+\left[1+2 f(x)+2 \sum_{m=0}^{M} h_{m}(x) \cos \left(2 \eta_{m} x-\gamma_{m}\right)\right] y=0,
$$

and let $\theta_{1}(x), \rho_{1}(x)$ belong to this equation as $\theta(x), \rho(x)$ in (1.11), (2.18) belong to (1.7). Then, the deduction of (2.22) shows that

$$
\lim _{x \rightarrow \infty} \rho_{1}(x)=+\infty \text { or exists (finite). }
$$

But $\theta(x) \equiv \theta_{1}(x)$ and $\rho_{1}(x) \equiv-\rho(x)$; thus $\rho(\infty) \neq+\infty$. Consequently, changing $c^{1},(2.17)$ becomes $r(x)=c^{1}+o(1), \rho(x) \equiv 0$.

Correspondingly, by the formulae following (2.19),

$$
\begin{aligned}
y_{j} & =\left[c_{j}^{1}+o(1)\right] \sin \left(c_{j}^{0}+o(1)+\theta(x)\right), \\
y_{j}^{\prime} & =\left[c_{j}^{1}+o(1)\right] \cos \left(c_{j}^{0}+o(1)+\theta(x)\right)
\end{aligned}
$$

and $c_{1}^{1} c_{2}^{1} \sin \left(c_{1}^{0}-c_{2}^{0}\right) \neq 0$. Thus, for a solution $y(x) \not \equiv 0, c^{1}>0$ in $r(x)=c^{1}$ $+o(1)$, and linearly independent solutions $y_{1}, y_{2}$ belong to pairs $\left(c_{1}^{0}, c_{1}^{1}\right),\left(c_{2}^{0}, c_{2}^{1}\right)$ with $c_{1}^{0} \neq c_{2}^{0}(\bmod \pi)$. This completes the proof of Theorem 1.2.

\section{REFERENCES}

1. F. V. Atkinson, The asymptotic solution of second order differential equations, Ann. Mat. Pura Appl. (4) 37 (1954), 347-378.

2. P. Hartman, On the zeros of solutions of second order linear differential equations, J. London Math. Soc. 27 (1952), 492-496.

3. R. B. Kelman and N. K. Madsen, Stable motions of the linear adiabatic oscillator, J. Math. Anal. Appl. (to appear).

The Johns Hopkins University 